\title{
Quelle unité pour l'argumentation? La concession, entre cellule et séquence argumentative
}

\author{
Thierry Herman \\ Universités de Neuchâtel et de Lausanne \\ Thierry.Herman@unine.ch
}

Résumé. La séquence argumentative de Jean-Michel Adam, inspirée du fameux modèle de Toulmin, peine à intégrer en son sein les concessions. Il est proposé ici de donner les contours d'une unité argumentative plus stable et plus flexible, la cellule, qui tiendrait compte des concessions et de voir comment cette unité permet de s'assembler à d'autres pour créer une séquence argumentative complexe.

\begin{abstract}
Which textual unit is suitable for dealing with argumentation?. Integrating concessive moves into Stephen Toulmin's layout of argument or Jean-Michel Adam's argumentative sequence is quite a challenge. This article aims to define a more flexible and stable argumentative unity: the argumentative cell - and attempts to study how cells combine to form a complete argumentative sequence.
\end{abstract}

\section{Introduction}

(1) Dominique Perret, skieur extrême: «Nous ne sommes pas des kamikazes suicidaires : certes, nous prenons des risques, mais ils sont mesurés ».

Cet exemple, souvent commenté par Jean-Michel Adam pour illustrer la séquence argumentative et les parenthésages (2011) illustre pourtant à mon sens un problème d'applicabilité de la séquence argumentative, largement inspirée par le modèle de Stephen Toulmin (1958). Il présente une unité de sens que l'on peut lier à la phrase graphique, de la majuscule au point, même si trois propositions la constituent, mais la conclusion du mouvement ( Nous ne sommes pas des kamikazes suicidaires ») occupe la première place de la phrase graphique alors que l'argument étayant cette conclusion est constitué par la troisième proposition. Le contre-argument, pris en "sandwich» entre les deux autres propositions, est donc parfaitement intégré à l'unité phrastique. Et pourtant, il n'est guère intégrable dans la séquence argumentative principale. C'est pourquoi Adam considère que le segment introduit par «certes» présente un argument ciblant une thèse antérieure contraire à la thèse défendue par le locuteur, une séquence argumentative enchâssée dans la séquence argumentative considérée.

Rappelons en effet que, selon Adam (2004 et 2011), le prototype de la séquence argumentative contient une macro-proposition 0 qui représente la thèse antérieure, puis des données-prémisses (P. arg 1), un étayage (p. arg 2) aboutissant probablement à une 
conclusion (nouvelle thèse) (P. arg. 3) sauf si une restriction (P. arg 4) invalide le mouvement. Cette modélisation de la séquence argumentative permet de représenter une forme de syllogisme étendu à plusieurs composantes ; mais, en intégrant la thèse antérieure, Adam passe de ce que Plantin appelle « une cellule argumentative » (2016:426) à une séquence argumentative contenant une contre-argumentation. Ainsi, dans le cadre de l'exemple (1), la reconstitution ne pose guère de problème pour les données ( $\mathrm{P}$. arg $1:$ : les risques sont mesurés ») et la conclusion ( $\mathrm{P}$ arg. $3:$ : nous ne sommes pas des kamikazes suicidaires »), la macro-proposition étayant ce mouvement étant implicite et faisant appel sans doute à une définition de sens commun sur le kamikaze comme prenant des risques non mesurés. Mais que faire de "nous prenons des risques »? La thèse antérieure de ce mouvement argumentatif est forcément non-p. arg 3, à savoir «Nous sommes des kamikazes suicidaires », thèse antérieure implicite, dont la nature d'antériorité peut d'ailleurs être discutable. L'argument étayant une telle conclusion est bien la deuxième proposition de la phrase graphique («nous prenons des risques »).

On pourrait en fait arguer une deuxième solution: considérer que «nous prenons des risques » est la P. arg 1 aboutissant à un P. arg 3 implicite (« Nous sommes des kamikazes suicidaires »), la restriction (P. arg 4) serait en ce cas « les risques sont mesurés », ce qui aboutit à une nouvelle conclusion : "Nous ne sommes pas des kamikazes suicidaires »). Il faut noter que la restriction mène en principe à une conclusion inverse à $\mathrm{P}$. arg 3 . Or, cette conclusion inverse n'est ni présente dans le prototype séquentiel d'Adam ni dans le modèle de Toulmin. Notons aussi que cette deuxième solution est contre-intuitive, dans la mesure où commencer une phrase par une conclusion inverse alors que l'on a des chances de considérer celle-ci comme le «claim » (au sens toulminien), le point de vue pris en charge par le skieur et l'aboutissement du raisonnement néglige le rôle de la prise de position du locuteur, élément phare du déploiement de l'argumentation.

Dans les deux cas, le problème théorique que cela pose est le suivant : a-t-on affaire à une ou deux unités argumentatives, sur le plan compositionnel ? Répondre à une telle question implique en premier lieu d'être au clair sur une définition de ce qui pourrait ressembler à une unité - ou une cellule - argumentative. A mon sens, l'intéressant ajout de Jean-Michel Adam au prototype toulminien, la thèse antérieure, ne suffit pas à résoudre totalement le problème. Une question de désignation fait déjà obstacle. En effet, la présentation d'une contre-argumentation comme une thèse antérieure ferait penser qu'un premier mouvement argumentatif est abouti, voire situé en contexte plus ou moins lointain, quand surgissent les macro-propositions de la séquence argumentative ; or, l'exemple (1) montre que l'unité de la phrase graphique intrique les deux mouvements. Ensuite, la désignation du mouvement exprimé par (1) comme une forme de séquence argumentative complète enchâssée dans une autre séquence pose un problème de cohérence avec l'idée exprimée dans l'intertitre de cette publication d'Adam (2004): «De la cellule argumentative à la séquence », où précisément, l'unité séquence est enrichie par la contreargumentation d'une autre cellule argumentative. En bref, il est difficile de savoir si l'unité argumentative minimale est la cellule ou la séquence, ou si la séquence doit contenir une thèse antérieure pour être nommée séquence argumentative. Dans cette communication, je défendrai la thèse que la "cellule argumentative» de Plantin constitue une unité de linguistique textuelle contenant plusieurs mouvements argumentatifs en opposition - dont certains peuvent être absents ou juste évoqués; et je suggère de parler de séquence argumentative pour une unité mésotextuelle constituée par un assemblage de cellules entre elles. Pour soutenir un tel propos, l'étude de la concession, qui s'inscrit mal dans le modèle toulminien, sera au centre de l'analyse. Après avoir présenté ma vision de la cellule argumentative, je présenterai une définition de la séquence argumentative complète, en passant par l'analyse d'un exemple complexe. 


\section{Le modèle toulminien et la question de l'autre dans le discours}

Il ne fait guère de doute que Stephen Toulmin est l'un des pères fondateurs du renouveau de l'étude de l'argumentation en situation concrète, à l'écart des principes et normes de la logique formelle. C'est pour cette raison que son modèle de l'unité transphrastique qu'est l'argumentation, le «layout of arguments », a intéressé bien au-delà du cercle des philosophes et des logiciens. On utilise en effet ce modèle dans le champ de la communication publique (van Eemeren et al. $2014: 228$ ), en linguistique textuelle (Adam 2011) ou en rhétorique et analyse de discours (de Jonge 2008). Malgré les critiques faites sur le caractère ambigu ou vague des propositions théoriques (van Eemeren 1996), les débats sur les catégories employées (Freeman, 2011; Hitchcock, 2003; Hitchcock \& Verheij, 2006), soixante ans après la première publication de son ouvrage, «The uses of argument ", la proposition de Toulmin reste une référence très souvent utilisée.

Rappelons en les principaux termes. Toulmin décrit son modèle en prenant soin de l'insérer dans une situation dialectique. Mettons qu'un locuteur exprime l'opinion $\mathrm{O}$, son interlocuteur pourrait ne pas l'accepter et exiger ce qui permet de fonder $\mathrm{O}$, poussant le premier locuteur à livrer des données (D) à la source de l'opinion $\mathrm{O}$ et donc de défendre $\mathrm{O}$. Il faut déjà relever ici deux problèmes liés au lexique, car cela a une incidence certaine sur la manière de percevoir la cellule argumentative en tant que telle. L'opinion défendue ${ }^{\mathrm{i}}$ est ma traduction de «claim », au sens d'un point de vue sur lequel le locuteur s'engage. Or, le traducteur de "The Uses of Argument» a choisi le lexème de "conclusion » (Toulmin 1993), choix malheureux à mon sens, puisqu'il fait référence au vocabulaire logique dont, précisément, Toulmin veut s'écarter en 1958. Dans «claim », l'idée de l'engagement du locuteur dans ce qu'il veut défendre est primordiale - cette ancre énonciative n'ayant aucune importance dans l'argumentation logique articulant prémisses et conclusion. Dans le même ordre d'idées, on peut relever que l'école amstellodamoise de la pragma-dialectique (van Eemeren \& Grootendorst 2004) a choisi le terme de «standpoint» et non de conclusion, qui semble, dans les travaux de langue anglaise, strictement cantonné à la logique. Le traducteur a aussi choisi le terme de « données » pour traduire « Data » (D), ce qui est de bon aloi en soi, mais il faut noter qu'ultérieurement, dans le manuel de Toulmin et al. (1979), le terme de "Data » est remplacé par un très suggestif "Grounds », qui renvoient plus au rôle de la proposition au sein d'une cellule argumentative qu'à la nature sémantique de ladite proposition; en effet, «Data » couvre uniquement dans l'ouvrage fondateur de Toulmin des propositions factuelles où les modalités aléthiques dominent. En remplaçant par des fondements ou des raisons, selon comment on choisit de traduire " grounds », la dimension transphrastique de l'argumentation apparaît plus nettement : il s'agit de produire les fondations, dans une ou plusieurs propositions, sur lesquelles l'opinion peut se construire.

Cette importante parenthèse étant terminée, je reviens au modèle : Toulmin présente la suite de la démarche dialectique de l'argumentation par une nouvelle question de l'interlocuteur, mettant au défi le responsable de $\mathrm{O}:$ : Comment en arrives-tu là ? » suscite une réponse sous la forme d'un Garant (W) («Warrant») articulant Données et Opinion défendue et soutenu par ce que l'on peut considérer comme une source évidentielle (cf. auteur 2015), le Backing ou support. Les doutes éventuels sur l'enchaînement sont exprimés par un «Qualifier » $(\mathrm{Q})$, un qualificateur modal permettant de faire varier le degré de certitude ou d'engagement par rapport à $\mathrm{O}$. Ce qualificateur modal ouvre la voie à une possible « restriction» («Rebuttal»), un contre-argument dont la propriété est d'annuler l'enchaînement argumentatif qui vient de se créer. L'exemple (2) peut s'analyser ainsi :

(2) On n'aura probablement $(\mathrm{Q})$ pas cours cet après-midi $(\mathrm{O})$. En effet, le secrétariat vient d'apprendre que le prof est malade (D). A moins qu'on ne trouve un remplaçant très rapidement $(\mathrm{R})$. 
Dans cet exemple, à l'instar du syllogisme logique qui est souvent exprimé sous la forme enthymématique, le Garant $(\mathrm{G})$ est implicite et fait référence à une pratique ancrée dans la réalité sociale du locuteur de 2, selon laquelle les cours sont en général annulés lorsque l'enseignant vient d'annoncer son absence. La restriction (R) se produit en raison même de la présence de $\mathrm{Q}$ : elle fait émerger une possibilité, dont on ne peut pas juger hors contexte la probabilité, d'un argument susceptible d'invalider le mouvement et de mener à l'opinion inverse : « on aura cours cet après-midi ».

La restriction permet ainsi d'ouvrir à une forme d'altérité argumentative; elle est intéressante dans la mesure où elle suggère une forme de développement du mouvement argumentatif en intégrant très clairement une dimension dialogique typique de l'argumentation dès lors qu'on la considère comme : "un mode spécifique de traitement verbal du désaccord, qui consiste en la construction de positions solides, c'est-à-dire étayées par un travail textuel de justification et situées par un travail interactionnel et dialogique de positionnement » (Jacquin et Micheli 2012, je souligne). Ce qu'il importe de relever, c'est que la restriction peut finalement être considérée comme une nouvelle Donnée, aboutissant à une nouvelle opinion (non-O), étayée par d'autres Garants, ouvrant peut-être une nouvelle Restriction et ainsi de suite. Et cela pose donc, fondamentalement la question de l'unité séquentielle.

Si une réflexion sur les modalités d'insertion d'une séquence argumentative dans une unité textuelle de rang supérieur ont été étudiées par (Herman \& Micheli 2015) en se fondant sur le fait que la linguistique textuelle envisage diverses possibilités d'agencement pluri-séquentiel au sein d'un texte (séquences coordonnées (succession), alternées (montage en parallèle) ou insérées (enchâssement)), celle-ci présuppose toutefois une capacité à définir ce que je pourrais appeler une unité argumentative de base. Cette unité, au centre de ma réflexion, doit recevoir les contours suffisants pour décrire et analyser de quelle manière elle permet de s'assembler entre elles au sein d'un mouvement mésotextuel : l'analogie avec le monde du vivant qu'exprime la «cellule argumentative» de Christian Plantin trouve ici son intérêt. Cette désignation illustre en effet l'idée de clôture d'un espace restreint qui est susceptible de se combiner avec des cellules voisines sur le plan cotextuel.

Or, l'exemple (2) pose un problème de fond : la restriction est-elle le point de départ d'une nouvelle argumentation? A quelle unité appartient-elle ? Poser cette question invite plus largement à repenser la question du désaccord au centre de l'argumentation et donc à la place que l'on accorde à l'autre dans l'argumentation monogérée. Ainsi, la concession antéposée que l'on trouve par exemple dans (3) peut-elle être considérée comme la donnée d'une séquence argumentative à laquelle s'oppose une autre donnée d'un second mouvement argumentatif? Dans l'exemple (4), la concession permet-elle d'envisager cet exemple comme une ou deux séquences argumentatives alors qu'aucun étayage n'est produit à propos de l'opinion défendue ?

(3) Ses basses manquent de profondeur, certes, mais elles sont audibles (http://www.lemonde.fr/pixels/article/2017/12/06/quelles-sont-les-meilleuresenceintes-bluetooth-a-offrir-en-cadeau-a-noel_5225724_4408996.html)

(4) Même si la majorité des abonnements s'effectue encore via des appareils mobiles et des ordinateurs portables, Netflix a bien conscience de cet attachement des consommateurs au confort du téléviseur. (https://france3regions.blog.francetvinfo.fr/regions-ftv/2017/12/20/revue-dactu-de-11h11mercredi-20-decembre-2017.html)

\section{Les contours de la cellule argumentative}

Dans les deux exemples (3) et (4), nous avons affaire à une phrase graphique. Comme pour les exemples précédents, il semble curieux que l'unité graphique établie par le scripteur 
puisse être décomposée en deux séquences argumentatives séparées et opposées entre elles. Le fait que cela représente un tout uniforme pour le scripteur est un premier argument qui invite à considérer que tous les exemples présentés forment une unité argumentative simple. En outre et à l'inverse de l'exemple (3), (4) peut se décliner avec une inversion des deux propositions-énoncés :

(4') Netflix a bien conscience de l'attachement des consommateurs au confort du téléviseur, même si la majorité des abonnements s'effectue encore via des appareils mobiles et des ordinateurs portables

Cette relative souplesse dans le déplacement des propositions concessives invite à penser que l'unité argumentative, la cellule argumentative donc, résiste, en tant qu'unité perceptible, à différents types de liage entre les composantes de cette cellule. Le fait de pouvoir indifféremment articuler «(a), car (b) » ou «(b) donc (a) » était déjà un indice que la séquentialité ou la linéarité langagière n'est pas un facteur déterminant par rapport aux rôles que les propositions-énoncés jouent au sein de la cellule argumentative. L'exemple (1) où le contre-argument est pris en sandwich entre l'opinion défendue et l'argument étayant ladite opinion montre un autre type d'arrangement, qui reste parfaitement compréhensible. Il est beaucoup plus ardu de considérer les exemples (5) et (6) comme formés d'une seule unité en revanche.

(5) Mideatik se présente comme une start-up. Toutefois, comme de nombreuses startups, le démarrage est lent. Cependant, elles misent sur un marketing ciblé grâce à Facebook. (http://defimedia.info/malheur-idee-et-projet-mideatik-unebouee-de-securite-pour-lete)

(6) Il n'est pas sûr que de telles conditions [interdire d'accéder au compte Facebook, Snapchat ou Instagram d'une personne décédée] soient autorisées. Mais comme le lieu de juridiction est aux Etats-Unis, le coût d'un procès serait donc très coûteux. Cependant, même le droit suisse ne laisse que peu de chances aux héritiers. (Le Temps, 17 décembre 2017)

La succession de deux connecteurs contre-argumentatifs articulant trois propositionsénoncés (toutefois/cependant ou mais/cependant) disloque la cellule argumentative, dans la mesure où l'opinion défendue par le locuteur ne l'est que provisoirement. Ainsi, on peut imaginer que la conclusion implicite de la première phrase de (5) est quelque chose comme "c'est une société prometteuse ", alors que la deuxième phrase mène à la conclusion inverse, l'opinion défendue par le locuteur, comme le veut la théorie de l'argumentation dans la langue selon laquelle l'argument suivant le «mais » est l'argument décisif. Or, la troisième phrase annule étrangement l'opinion défendue pour défendre à nouveau la conclusion de la première proposition - «c'est une société prometteuse », ce qui représente un effort cognitif considérable dans lequel on peine à «fixer» l'opinion finalement défendue par le locuteur.

Ces exemples suggèrent en creux que le noyau de la cellule argumentative - pour continuer l'analogie - est bien l'opinion (provisoirement) défendue. C'est à mes yeux la possibilité d'isoler l'opinion défendue qui donne les contours de l'unité argumentative, dans laquelle on peut trouver une cellule simple ou complexe. D'ailleurs, lorsque Toulmin présente son modèle, il me semble significatif qu'il commence par elle : le «claim ». Il ressort de là 1 . que les concessions, qui étayent faiblement un point de vue contraire à l'opinion défendue, ne sont lisibles qu'en regard du «claim » et intègrent, de fait, la même unité de la cellule argumentative 2. que la présence d'une nouvelle opinion défendue par le locuteur implique que l'on change de cellule argumentative.

Cette double proposition conduit à considérer que la séquence argumentative doit intégrer, de manière non-nécessaire, différentes formes de contestation possible de l'opinion défendue sans considérer pour autant que cette intégration passe par un enchâssement. On peut donc intégrer une forme de contestation faible - les concessions - et 
une forme de contestation forte - les restrictions. Autrement dit, la proposition consiste à défendre l'idée que la cellule argumentative peut intégrer jusqu'à trois schémas toulminiens $^{i i}$ en son sein sans changement d'unité compositionnelle (cf. un premier essai de typologie dans Oswald et Herman 2016). L'exemple suivant, un commentaire d'une photo d'un plat sur TripAdvisor, illustre une cellule argumentative de ce type :

(7) «Beaux mais trop sucrés, sauf si on apprécie les bonbons en fin de repas !» (https://www.tripadvisor.fr/LocationPhotoDirectLink-g187148-d1086705-

i231979580-La_Veranda_by_Gordon_Ramsay-

Versailles_Yvelines_Ile_de_France.html)

Dans cet exemple, «beaux» est un argument qui pousse à inciter les voyageurs du restaurant à commander ce plat, le garant implicite étant qu'un beau plat invite à être mangé, « mais trop sucrés » est un argument qui mène à l'opinion défendue par le locuteur, consistant plutôt à déconseiller de commander ce plat selon un Garant implicite considérant que les plats trop sucrés ne sont pas agréables à manger. Enfin, «sauf si on apprécie les bonbons en fin de repas » est une restriction qui, le cas échéant, conseille quand même de commander ce plat selon un troisième Garant implicite selon lequel les plats aussi sucrés qu'un bonbon peuvent plaire à certains individus, .

A la différence des exemples (5) et (6), l'opinion défendue par le locuteur reste stable est vise plutôt à déconseiller le plat tout en laissant l'exception ouverte, exception qui annulerait, pour les personnes concernées, la recommandation du locuteur. On voit en outre que l'unicité de cette cellule correspond à l'unique phrase graphique constituant la légende de la photo. Cette correspondance entre unités phrastiques et argumentatives vaut pour les exemples (1), (3) et (4) qui contiennent pourtant des contre-arguments. Je veux dire par là que la redéfinition de la cellule argumentative telle qu'elle est proposée ici permet de mieux rendre compte, avec le principe du rasoir d'Ockham, les problèmes d'ajustement entre unité graphique (la phrase allant de la majuscule au point) et unité argumentative lorsqu'une concession est intégrée dans des phrases graphiques. La stabilité du noyau est le critère permettant de fonder une cellule argumentative maximale qui se présente dès lors selon la forme suivante :

ZONE CONCESSIVE

Argument(s) concédé(s) MAIS DONC

Opinion autre
ZONE ARGUMENTATIVE ZONE EXCEPTIVE Argument(s) pris en charge SAUF SI Exception(s) DONC Opinion défendue
DONC

Opinion autre

Fig. 1. La cellule argumentative maximale

\section{Les différentes formes de cellules argumentatives}

Un tel dispositif n'est pas totalement inconnu. En français, deux options sont généralement mises en évidence lorsque l'on évoque des concessions, l'option ternaire de concession logique/concession argumentative et concession rectificative (Morel 1996) ou l'option binaire de Moeschler entre concession logique et concession argumentative.

Or, dans son ouvrage de 1985, Jacques Moeschler présentait les triangles concessifs et le carré argumentatif en tenant compte des différents pôles que reliaient les connecteurs. Le carré argumentatif est décrit ainsi et correspond à la zone concessive et zone argumentative du schéma précédent : 


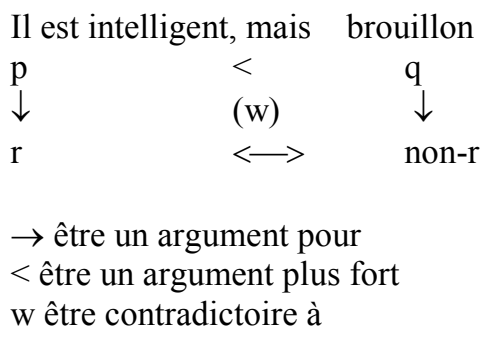

Fig. 2. Le carré argumentatif de Moeschler (1985)

De manière significative, avec de Spengler, il affirmait en 1982 :

Ce carré argumentatif est d'une part propre à la concession, mais d'autre part permet de définir de plus près la notion d'argumentation. A ce titre, nous dirons qu'une argumentation s'inscrit toujours dans le cadre d'un débat contradictoire. En d'autres termes, une argumentation présuppose d'une part une finalité du discours (on parle pour défendre une certaine thèse) et d'autre part une opposition discursive (on défend certaines thèses par opposition à d'autres thèses contraires ou contradictoires). (De Spengler et Moeschler 1982 : 14).

C'est en se fondant sur cette définition que les auteurs distinguent la concession logique du type "Bien que la rivière fût en crue, le pont ne s'est pas écroulé » de la concession argumentative. Sans entrer dans les débats sur la nature et le type de concession (cf. par exemple Garnier et Sitri 2008), il m'importe de souligner ici que la notion de thèse (proche de l'opinion défendue ${ }^{\mathrm{iii}}$ ) implique une prise en charge énonciative, exprimée par des modalités épistémiques, appréciatives, bouliques, etc. - à l'exclusion des modalités aléthiques que l'énoncé exemplaire de la concession logique ci-dessus exprime (ainsi que de certaines modalités épistémiques, voir Herman 2015 sur ce point). La concession dite logique s'inscrit plutôt dans une démarche explicative que dans une dimension argumentative, dans laquelle l'implication causale est déterminante. Il n'est à mon sens pas possible de considérer ici que l'on a affaire à une séquence argumentative, quand bien même les structurations et relations interphrastiques sont singulièrement proches de l'argumentation.

En revanche, un autre exemple classique comme «Bien qu'il pleuve, je sors » pourra, en raison de l'action prise en charge par le locuteur, être considéré comme une séquence argumentative incomplète. Le «carré argumentatif » laisse une place vacante, mais pas forcément inexistante, pour les arguments motivant l'opinion défendue par le locuteur. En effet, le ou les arguments pour "sortir" sont absents, mais la structure concessive ellemême implique qu'il existe une ou plusieurs raisons plus fortes que la pluie pour décider de sortir. Un interlocuteur pourrait être mis au défi de justifier une telle décision, en somme de demander les «données » à l'appui de l'opinion défendue. Evidemment, sans la structure concessive, un énoncé comme « je sors » ne pourrait plus être considéré comme une forme d'argumentation. Je considère donc que la co-présence d'une opinion défendue et d'un étayage, même si ce dernier ne concerne que l'opinion non-prise en charge par le locuteur, permet de faire entrer cette suite de propositions dans un mouvement argumentatif ${ }^{\text {iv }}$.

Le modèle ternaire de Morel (1996) introduit une concession rectificative, exprimée par les exemples suivants :

(8) J'irai aux USA le mois prochain - encore que j'irai pas - parce qu'il y aura l'affaire d'EDF. (Morel 1996 : 11)

(9) (a) Difficile de trouver des lieux coupés du réseau social, (b) hormis la très rase campagne et les sommets. (c) Encore que: l'aiguille du Midi résonne de nombreux gazouillis et ce ne sont pas ceux du gypaète barbu. (Le Temps, 20 décembre 2014)

Dans de tels exemples, on trouve deux opinions défendues par le même locuteur qui annule de son propre chef son engagement dans la première opinion. C'est un cas 
problématique pour la cellule argumentative : en effet, la définition que l'on propose plus haut du noyau de la cellule argumentative implique que la concession rectificative, qui modifie l'opinion venant d'être défendue par le locuteur, ouvre une nouvelle unité argumentative. De fait, la ponctuation semble mettre en scène l'interruption brusque (exemple 8) ou la fin (9) d'une unité avant un «rebondissement» introduit par «Encore que ». C'est pourquoi on ne peut plus parler d'une seule séquence argumentative, mais bien de deux unités. Si on veut, le "locuteur d'après » "encore que » se désolidarise du " locuteur d'avant », même si c'est la même personne, et ce, parfois radicalement comme l'illustre l'exemple (8).

Mais l'affaire est plus corsée dans l'exemple (9). En effet, dans ce billet d'humeur commentant l'usage intensif de Twitter dans n'importe quel environnement, la concession rectificative (c) vient en fait ironiquement renforcer l'opinion défendue (a) : l'exception « Aiguille du Midi » aux exceptions formulées et prises en charge par le locuteur en (b) ne fait que renforcer l'impression que le réseau social se propage sur toute la planète. On pourrait considérer sur le plan de la compositionnalité que (b) «hormis la très rase et campagne et les sommets » est une concession que la concession rectificative introduite par « encore que » réfute. Dans tous les cas, on a bien affaire à deux séquences argumentatives, qui en fait pointent vers une même conclusion (a), laquelle est en quelque sorte identique à l'opinion vers laquelle pointe la rectification (c). Alors que (8) présente une remise en question de l'opinion, (9) remet (au moins partiellement) en question les concessions que le locuteur voulait bien faire, ce qui, finalement, renforce l'opinion défendue en (a).

\section{Méso-textualité : l'assemblage de cellules argumentatives}

Dans le modèle de la cellule argumentative défendue ici, les possibilités d'assemblages entre cellules se font selon trois directions : 1. Par le haut : les données étayant une opinion sont elles-mêmes étayées (ex. 10) ; 2. Par le bas, l'opinion défendue devient une donnée pour une nouvelle opinion défendue, et ce, jusqu'à la thèse générale (ex. 11);3. Par la droite: une rectification ou une restriction à une restriction vient annuler la première opinion défendue (ex. 5 et 6 ).

Pour définir une séquence argumentative composée de différentes cellules, on peut envisager les bornes initiales et finale de la séquence. Du côté des bornes initiales, on cherchera une donnée faisant office de point de départ de l'argumentation, non étayée et posée comme partagée, zone de l'accord préalable cher à Perelman et Olbrechts-Tyteca (1958). Du côté de la borne finale, on dira que tant qu'un élément composant une cellule argumentative est réutilisé dans une autre cellule argumentative avec un autre rôle, ces cellules s'assemblent entre elles. Autrement dit, si, par exemple, l'opinion défendue (rôle 1) sert de donnée (rôle 2) à une nouvelle opinion défendue, ce nouveau rôle dans le chaînage indique le développement d'un mouvement argumentatif non terminé.

(10)(a) Dans la pratique, aucune devise ne pourrait remplacer aujourd'hui le billet vert en tant que monnaie de réserve et d'échange. (...) (b) Placer des réserves en euros reste délicat, (c) les solidités respectives des créances grecques, irlandaises, italiennes ou allemandes étant jugées très différemment.

(11)(a) En outre, l'économie américaine pourrait surprendre par sa vigueur l'an prochain, (b) le plan de relance massif de Washington déployant pleinement ses effets. (c) Le billet vert apparaîtrait alors sous un jour plus favorable.

L'exemple (10) offre une structure subordonnée de type : (a) [en effet] ${ }^{\mathrm{v}}$ (b) [en effet] (c) tandis que l'exemple (11) présente une structure de type : $\{(\mathrm{a})$ en effet (b) $\}$ (donc) (c) que l'on peut reconstruire par: (b) [donc] (a) [donc] (c). Cette construction de chaînages mésotextuels conduit à plusieurs observations. La première est liée à la théorie anglosaxonne de l'argumentation qui considère trois structures complexes d'argumentation: 
l'argumentation convergente, l'argumentation liée et l'argumentation subordonnée (Freeeman 2011, Snoek Henkemans 1992, Walton 1996). Alors que les deux premières observent en fait de quelle manière les données s'assemblent entre elles pour aboutir à la même conclusion (intracellulaire), la dernière est typiquement un chaînage par le haut ou par le bas et sort de la cellule argumentative proprement dite pour intégrer une dimension mésotextuelle.

La deuxième observation est que, comme l'argumentation peut être aussi bien progressive (A car B) que régressive (B donc $\mathrm{A}$ ), la reconstitution du chaînage vise plus une reconstitution d'un raisonnement que la description d'un mouvement textuel : on voit que l'exemple (11) présente sur le plan de la linéarité langagière un mouvement régressif (a [en effet] b) suivi d'un mouvement progressif (a [donc] c) que l'on reconstituera sous une forme entièrement progressive par (b [donc] a [donc] c), afin de dégager la borne de début et de fin de la séquence argumentative. Il faudra faire de même avec les concessions : les exemples (4) et (4') recevront ainsi le même schéma indépendamment du moment d'apparition du segment marqué par "même si ». Quand bien même la linguistique textuelle est une discipline descriptive, il me paraît important de dégager, en analysant des structures d'argumentation, les étapes du raisonnement entre les premières données et la thèse ultime, ce qui implique une légère reconstruction par rapport à la linéarité textuelle.

\section{Etude d'un cas}

Observons un cas réel de construction d'une séquence argumentative complète très complexe, combinant plusieurs cellules argumentatives - un éditorial du Temps qui s'interroge sur la puissance du dollar en tant que monnaie de référence mondiale, d'où sont tirés les exemples (10) et (11).

(a) En outre, l'économie américaine pourrait surprendre par sa vigueur l'an prochain, (b) le plan de relance massif de Washington déployant pleinement ses effets. (c) Le billet vert apparaîtrait alors sous un jour plus favorable. (d) Même si la question de l'endettement des Etats-Unis et du risque inflationniste ne serait pas résolue pour autant.

(e) Certes, la montée en puissance des économies émergentes dans le sillage de la Chine change la donne. (f) Mais le consommateur de dernier ressort reste la famille américaine, $(\mathrm{g})$ même si son excès d'endettement donne lieu à un sevrage pénible.

(h) De plus, les Etats-Unis resteront encore longtemps la première puissance économique et militaire. (i) Ils savent trop bien quel avantage il y a à voir sa devise disposer d'un statut de référence: (j) Washington peut s'endetter librement et copieusement dans sa propre monnaie, $(\mathrm{k})$ et a la possibilité d'en imprimer à volonté. (Jean-Pascal Baechler, Le Temps, 22 octobre 2009)

Dans cet extrait, on peut voir à mon sens quatre cellules argumentatives assemblées en une séquence - je mets ici en gras les opinions défendues, noyaux des cellules considérées :

1. (b) [donc] (a)

2. Certes (e) + Même si (g), (f)

3. $(\mathrm{i} / \mathrm{j} / \mathrm{k})^{\mathrm{vi}}[$ donc $](\mathbf{h})$

4. Même si (d), (a) + (f) + (h) [donc] (c)

La thèse de l'extrait est donc « le billet vert apparaîtra sous un jour plus favorable ». A l'appui de cette thèse, trois arguments : les effets du plan de relance massif, la famille américaine comme consommateur de référence, les USA durablement première puissance économique et militaire. On trouve également trois arguments concédés : l'endettement des Etats-Unis est une concession par rapport à la thèse, la montée des puissances émergentes ainsi que l'excès d'endettement sont des concessions par rapport à l'argument de la famille 
américaine comme source de référence. On voit enfin un certain nombre de propositions qui sont posées comme partagées ou suscitant l'accord - car elles ne font pas l'objet d'une justification: (b), (d), (e), (f), (g), (i/j/k). Ce nombre important de points de départ argumentatifs censés être partagés peut s'expliquer par le genre de discours : brièveté de l'éditorial, locuteur porte-parole de la doxa (cf. Herman et Jufer 2001).

Dans tous les cas, l'outil proposé ici permet d'exercer une analyse tabulaire et structurelle d'un texte argumentatif en mettant en évidence des chaînages entre propositions-énoncés. Il permet également de rendre compte avec efficacité, me semble-t-il, du poids des arguments et des contre-arguments, de dégager des zones de faiblesse tant du point de vue rédactionnel (ainsi la structure en boucle de «certes (e), mais (f), même si (g) » pourrait être simplifiée par «Même si (e) et (g), (f) ») que du point de vue de la résistance à la contestation (Doury 2016) : ainsi, l'argument (f), non-étayé et doublement concédé, pourrait paraître assez dogmatique et nécessitant un renforcement argumentatif.

$\mathrm{Si}$ on traduisait notre analyse par un schéma ${ }^{\text {vii }}$ dans lequel on lit l'argumentation de haut en bas et dans lequel on tente de garder en partie gauche les contre-arguments et en partie droite les exceptions, cela donnerait $\operatorname{ceci}^{\mathrm{viii}}$ :

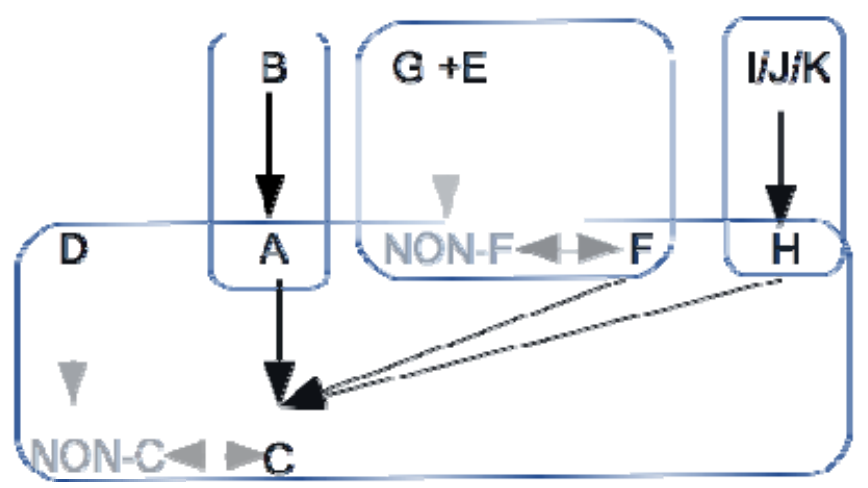

Fig. 3. Schéma structurel de la séquence argumentative analysée

\section{Conclusion : les avantages d'une cellule redéfinie}

Définir ainsi cellule argumentative et séquence argumentative permet de clarifier un point complexe pour décrire des réalités langagières. En effet, on se dote avec la cellule argumentative d'une unité stable, qui, dans un processus bottom-up, s'assemble au sein d'un mouvement mésotextuel appelé ici séquence. Cette désignation de séquence en écho aux travaux de Jean-Michel Adam est sans doute discutable - on aurait pu parler de plan de texte ou de mouvement argumentatif - mais l'idée que l'on retrouve finalement des données (les points de départ non-étayés), une thèse finale (l'ultime opinion défendue), des formes d'altérité de voix exprimées par des concessions et des restrictions est très proche $\mathrm{du}$ prototype séquentiel argumentatif, même s'il peut contenir tout un chaînage d'argumentations subordonnées.

D'un point de vue pédagogique, la difficulté de lecture que posent des textes argumentatifs peut être réduite par un outil qui est la description schématique d'un raisonnement tel qu'il est déployé dans un texte. Et l'unité désignée permet d'appréhender avec plus de simplicité la dimension concessive des structures argumentatives sans forcément disloquer l'unité scripturale qu'est la phrase graphique et sans passer par une thèse antérieure qui est la solution imaginée par Adam. 
Stabilité et allègement ne doivent pas pour autant dissimuler les problèmes que posent les reconstructions d'argumentation, tant les textes que l'on analyse peuvent être parfois surprenants, redondants ou proposer des sauts dans le raisonnement. L'extrait analysé ici est plus ou moins clair - je le donne à mes étudiants comme exercice de reconstruction mais il a été choisi pour cette raison. Les relations de discours qui touchent à l'argumentation sont loin de passer que par les connecteurs. Par exemple, les verbes de causalité peuvent exprimer une relation d'argument entre le sujet et le prédicat alors que l'on a non seulement une unité graphique, mais aussi grammaticale. Ce problème mérite toutefois d'autres développements. L'enjeu de cette communication était de poser une base aussi résistante que possible pour décrire l'argumentation du point de vue de la linguistique textuelle.

\section{Références bibliographiques}

Adam, J-M. (2004), Une approche textuelle de l'argumentation: "schéma", séquence et phrase périodique, in M. Doury et S. Moirand (éds.), L'argumentation aujourd'hui. Positions théoriques en confrontation. Paris : Presses de la Sorbonne Nouvelle, 77-102.

- (2011), Les textes : types et prototypes, Paris : Armand Colin.

Doury, M. (2016). Argumentation. Analyser textes et discours. Paris : Colin.

Freeman, J. B. (2011). Argument Structure: Representation and Theory, London: Springer.

Garnier, S. \& Sitri, F. (2008). Problèmes épistémologiques en analyse du discours : le cas de l'énoncé concessif. In. Homenagem a Michel Pêcheux. 25 anos de presença na analise do discurso, Mercado de Letras (Campinas, Brésil), 99-106

Herman, T. \& N. Jufer (2001). «L'éditorial, "vitrine idéologique du journal" ?», Semen n¹3, Besançon, Université de Franche-Comté

Herman, T. (2015). A plea for a linguistic distinction between explanation and argument. In F. van Eemeren, \& Garssens, B. Reflections on Theoretical Issues in Argumentation Theory, Amsterdam: Springer, 247-258.

Herman, T. \& R. Micheli (2015). Théorie de l'argumentation et théorie du texte : quelques enjeux d'une approche textuelle de l'argumentation, in J.-M. Adam \& al., Faire texte, Presses universitaires de Franche-Comté, 81-110.

Hitchcock, D. (2003). Toulmin's warrants. In F. H. V. Eemeren, J. A. Blair, C. A. Willard, \& A. F. S. Henkemans (Eds.), Anyone who has a view, 69-82.

Hitchcock, D., \& Verheij, B. (Eds.), (2006). Arguing on the Toulmin Model. Dordrecht: Springer Netherlands.

Jacquin, J. et R. Micheli, (2012). « Entre texte et interaction : propositions méthodologiques pour une approche discursive de l'argumentation en sciences du langage ». In Frank Neveu et al. (éds.), Actes $d u \quad 3^{\text {ème }}$ Congrès mondial de linguistique française, [en ligne], http://www.linguistiquefrancaise.org/

De Jonge, E. (2008). Pertinence de l'utilisation du modèle de Toulmin dans l'analyse de corpus. Argumentation \& Analyse du discours, ${ }^{\circ} 1$, [en ligne], http://aad.revues.org/251

Moeschler, J. et De Spengler, N. (1982). La concession ou la réfutation interdite: approches argumentative et conversationnelle. Cahiers de linguistique française, 4, 7-36.

Moeschler, J., (1985), Argumentation et conversation : éléments pour une analyse pragmatique du discours. Paris : Hatier.

Morel, M-A. (1996). La concession en français, Gap : Orphys.

Oswald, S. \& Herman T. (2016), Argumentation, conspiracy and the Moon: a rhetoric-pragmatic analysis. In M. Danesi, \& S. Greco, Case Studies in Discourse Analysis. Munich: Lincom Europa, 295-330.

Perelman, C, \& Olbrechts-Tyteca, L., (1958). Traité de l'argumentation: La nouvelle rhétorique. Paris: Presses univ. de France.

Plantin, C., (1990) Essais sur l'argumentation, Paris : Kimé.

— (1996) L'argumentation, Paris : Seuil, coll. « Mémo ».

- (2016) Dictionnaire de l'argumentation, ENS Lyon.

Snoek Henkemans, F., (1992). Analysing complex argumentation, Amsterdam : SicSat. 
- (2000) State-of-the-art: The Structure of Argumentation. Argumentation. An international journal on reasoning, $\mathrm{n}^{\circ} 14,447-473$.

Toulmin, S.E., (1958 (1993, trad. fr.)). Les usages de l'argumentation. Paris : PUF.

Toulmin, S.E., Rieke, R. D., \& Janik, A. S. (1979). An introduction to reasoning. New York: London: Macmillan; Collier Macmillan.

Van Eemeren, F. (1996). Fundamentals of argumentation theory: A handbook of historical backgrounds and contemporary developments. Mahwah NJ: L. Erlbaum.

Van Eemeren, F. et R. Grootendorst, (2004). A Systematic Theory of Argumentation. The PragmaDialectical Approach. Cambridge : CUP.

Van Eemeren, F. \& al. (2014). Handbook of argumentation theory. Dordrecht: Springer.

Walton, D. (1996). Argument Structure. A Pragmatic Theory, Toronto : University of Toronto Press.

${ }^{\mathrm{i}}$ Je distingue l'opinion - unité propositionnelle analysable en soi que je sépare de l'assertion factuelle par la forme d'engagement du locuteur dans son dire - de l'opinion défendue, qui engage une dimension de relation entre des unités de type clause ou propositions-énoncés (Adam 2011).

ii Plus précisément, il s'agit de trois schémas sans la «restriction » / Rebuttal, qui prend une place à part dans la proposition faite ici (voir plus bas).

iii J'utilise le terme de thèse pour évoquer l'opinion finale d'un développement argumentatif complexe au sein d'une structure mésotextuelle (voir plus loin).

${ }^{\text {iv }}$ Il faut donc noter que l'exemple «Bien qu'il pleuve, la route est sèche », parce qu'il ne présente pas une opinion défendue, n'offre plus qu'une concession logique. Le locuteur n'a pas à expliquer le fait constaté (i.e. la route sèche) : il peut se contenter de constater l'étrangeté de la situation sans être en position de devoir offrir une explication, ce qui fait la différence essentielle avec la cellule argumentative.

${ }^{v}$ Je mets entre crochets des connecteurs prototypiques du mouvement régressif (en effet), progressif (donc) ou oppositif (mais) lorsqu'ils sont absents. Je marque aussi par des accolades un enchaînement argument-opinion qu'il faut considérer dans son ensemble plutôt que l'un des segments (argument ou conclusion). L'absence de marques de connexion explicite est un problème que l'on peut résoudre par les travaux sur les relations de discours, mais je ne m'arrêterai pas à cette question dans cette publication.

${ }^{v i}(\mathrm{j})$ et (k) élaborent à mon sens l'avantage mentionné en (i) : je ne vois pas de relation d'étayage dans le deux-points ici.

${ }^{\text {vii }}$ Il existe de nombreux travaux sur la représentation graphique de l'argumentation. La proposition faite ici est une tentative qui pourrait être affinée dans une publication ultérieure.

viii Je ne représente pas les Garants, les Supports ou les Qualificateurs modaux de la cellule, afin de ne pas charger le schéma. 